\title{
SAC-CI Study on the Excited and Ionized States of Free-Base Porphin: Rydberg Excited States and Effect of Polarization and Rydberg Functions
}

\author{
Y. Tokita, ${ }^{\dagger}, \sharp \S$ J. Hasegawa, ${ }^{\dagger}$ and H. Nakatsuji ${ }^{*},+,, \|$, \\ Department of Synthetic Chemistry and Biological Chemistry, Faculty of Engineering, Kyoto University, \\ Sakyo-ku, Kyoto 606-01, Japan, The Institute for Fundamental Chemistry, 34-4 Takano Nishi-Hiraki-cho, \\ Sakyo-ku, Kyoto 606, Japan, Research Center, Denki Kagaku Kogyo Co., Ltd., 3-5-1 Asahimachi, \\ Machida-shi Tokyo 194, Japan, and Department of Applied Chemistry, Graduate School of Engineering, \\ The University of Tokyo, Hongo, Tokyo 113, Japan
}

Received: September 26, 1997; In Final Form: December 17, 1997

The ground, excited, and ionized states of free-base porphin (FBP) are reinvestigated by the SAC (symmetryadapted cluster)/SAC-CI (configuration interaction) method. In particular, the Rydberg excited states, the valence-Rydberg mixing, and the effect of polarization d-functions are studied. The lowest Rydberg excited state would exist at around $5.5 \mathrm{eV}$ with a weak intensity, but the valence-Rydberg mixing is small. The effects of both the Rydberg and polarization d-functions are relatively small for the excitation energies of the main peaks, whereas the polarization d-functions affect considerably the ionization spectrum.

\section{Introduction}

Porphyrins have important functions in biological systems. ${ }^{1}$ To elucidate the mechanisms of these functions, the electronic structures of porphyrins have been actively investigated. ${ }^{2-25}$ The excited and ionized states of porphyrins observed in the $\mathrm{VUV}^{26}$ and photoelectron spectra, ${ }^{27}$ respectively, have been investigated by semiempirical ${ }^{2-8}$ and ab initio methods. ${ }^{9-13}$ In our laboratory, the ground, excited, and ionized states of many kinds of porphyrins have been investigated ${ }^{14-20}$ by the SAC (symmetry-adapted cluster ${ }^{28} / \mathrm{SAC}-\mathrm{CI}$ (configuration interaction) ${ }^{29,30}$ method. Among these, free-base porphin (FBP) shown in Figure 1 is a key compound, and the result was as follows. ${ }^{14}$ (1) The ground state could be described by the single-reference (Hartree-Fock configuration) theory. (2) The excitation energy and oscillator strength reproduced well the experimental spectrum, ${ }^{26}$ and new assignments for the $\mathrm{B}, \mathrm{N}, \mathrm{L}$, and $\mathrm{M}$ bands were proposed. (3) Although Gouterman's four-orbital model ${ }^{1}$ appeared to be valid for the $\mathrm{Q}$ bands, it broke down for the $\mathrm{B}$ and $\mathrm{N}$ bands because the excitations from the lower $\mathrm{b}_{1 \mathrm{u}}$ orbital mixed strongly with these bands. Further, the mixing was different for the B and $\mathrm{N}$ bands. (4) Mixing of $\sigma-\sigma^{*}$ excitations with $\pi-\pi^{*}$ excitations was shown to be important, particularly in the $2^{1} \mathrm{~B}_{3 \mathrm{u}}(\mathrm{B}), 2^{1} \mathrm{~B}_{2 \mathrm{u}}(\mathrm{N})$, and $3^{1} \mathrm{~B}_{3 \mathrm{u}}(\mathrm{L})$ states. (5) The calculated ionization spectrum roughly explained the Dupuis photoelectron spectrum. ${ }^{27}$ The effects of the reorganizations and electron correlations were large particularly for the ionizations from the n and $\sigma$ orbitals.

These results as a whole reproduced well the electronic features of FBP. However, when we performed such calculations, the computational environment was not good: we had to use $2 p$ double- $\zeta$ basis sets and a relatively small active space. Therefore, in this study we perform two higher-level calculations of FBP to elucidate the accuracy of the previous calculations.

\footnotetext{
* To whom correspondence should be addressed. Fax: 81-75-753-5910.

$\pitchfork$ Kyoto University.

The Institute for Fundamental Chemistry.

$\S$ Denki Kagaku Kogyo Co., Ltd.

"The University of Tokyo.
}

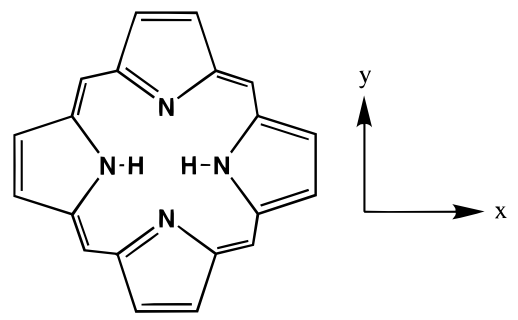

Figure 1. Structure of free-base porphin.

One calculation uses the valence double- $\zeta$ basis set plus Rydberg functions to clarify the Rydberg excited states and the effect of the Rydberg functions on the excited states. Since the peaks of the Rydberg excited states are generally weak,,$^{31-33}$ the assignment of the Rydberg excited states is difficult by experimental technique alone and theoretical information is necessary. Furthermore, if the valence-Rydberg mixing is large as in the excited states of ethylene, ${ }^{31}$ furan,${ }^{32}$ benzene, ${ }^{33}$ etc.,${ }^{34}$ good results cannot be expected by the calculations including valence basis sets alone, and a large basis set dependence may exist. ${ }^{31-33}$ The other calculations use the valence double- $\zeta$ basis set plus polarization d-functions to improve the description of the valence excited and ionized states. ${ }^{33}$ Both calculations are carried out using a larger active space than that in the previous calculation. The details of the calculations are described in the next section. The accuracy and effectiveness of the SAC/SACCI method are now well established, ${ }^{35,36}$ even for a relatively large porphyrin group of molecules.

\section{Methods}

FBP $\mathrm{C}_{20} \mathrm{~N}_{4} \mathrm{H}_{14}$ is assumed to have $D_{2 h}$ symmetry with a $D_{4 h}$ skeleton (Figure 1). The atomic coordinates taken from Sekino and $\mathrm{Kobayashi}^{7}$ are the same as the previous one for comparison with our previous result. This molecule consists of 162 electrons and 38 atoms.

We performed two different calculations, $\mathrm{A}$ and B. In calculation $\mathrm{A}$, the basis set is of double- $\zeta$ quality for the valence 
TABLE 1: Dimensions of the Linked Terms for SAC/SAC-CI Calculations A, B, and C of the Singlet States, and for Calculations B and $C$ of the Ionized States of Free-Base Porphin

\begin{tabular}{|c|c|c|c|c|c|c|c|c|c|c|c|}
\hline \multirow{5}{*}{$\begin{array}{c}\text { state } \\
(\mathrm{r}-\text { nos. })^{a}\end{array}$} & \multicolumn{7}{|c|}{ singlet state } & \multicolumn{4}{|c|}{ ionized state } \\
\hline & \multicolumn{5}{|c|}{ calculations } & \multirow{2}{*}{\multicolumn{2}{|c|}{ C }} & \multicolumn{4}{|c|}{ calculations } \\
\hline & \multicolumn{2}{|c|}{ B } & \multicolumn{3}{|c|}{ A } & & & \multicolumn{2}{|c|}{ B } & \multicolumn{2}{|c|}{$\mathrm{C}$} \\
\hline & \multirow{2}{*}{$\begin{array}{c}\text { before } \\
\text { selection }\end{array}$} & \multirow{2}{*}{$\begin{array}{c}\text { after } \\
\text { selection }\end{array}$} & \multirow{2}{*}{$\begin{array}{c}\text { before } \\
\text { selection }\end{array}$} & \multicolumn{2}{|c|}{ after selection } & \multirow{2}{*}{$\begin{array}{c}\text { before } \\
\text { selection }\end{array}$} & \multirow{2}{*}{$\begin{array}{c}\text { after } \\
\text { selection }\end{array}$} & \multirow{2}{*}{$\begin{array}{c}\text { before } \\
\text { selection }\end{array}$} & \multirow{2}{*}{$\begin{array}{c}\text { after } \\
\text { selection }\end{array}$} & \multirow{2}{*}{$\begin{array}{l}\text { before } \\
\text { selection }\end{array}$} & \multirow{2}{*}{$\begin{array}{c}\text { after } \\
\text { selection }\end{array}$} \\
\hline & & & & valence & Rydberg & & & & & & \\
\hline \multicolumn{12}{|l|}{ SAC } \\
\hline $\mathrm{A}_{\mathrm{g}}(1)$ & 17879553 & 84928 & 4410565 & 11478 & & 1362631 & 10879 & & & & \\
\hline \multicolumn{12}{|l|}{ SAC-CI } \\
\hline $\mathrm{A}_{\mathrm{g}}(2,8)$ & 17879553 & 158339 & & & & 1362631 & $46199^{b}$ & 132208 & 65380 & 25560 & 6939 \\
\hline $\mathrm{B}_{1 \mathrm{~g}}(3,7)$ & 17871092 & 239449 & & & & 1360246 & 54848 & 132105 & 62359 & 25523 & 5985 \\
\hline $\mathrm{B}_{2 \mathrm{~g}}(1,3)$ & 16052930 & 119097 & & & & 1308922 & 21230 & 102610 & 28938 & 22979 & 3082 \\
\hline $\mathrm{B}_{3 \mathrm{~g}}(1,3)$ & 16052830 & 120767 & & & & 1308922 & 21441 & 102586 & 27971 & 22979 & 2809 \\
\hline $\mathrm{A}_{\mathrm{u}}(1,2)$ & 16052770 & 118294 & & & & 1308888 & 20688 & 102648 & 37431 & 22962 & 1755 \\
\hline $\mathrm{B}_{1 \mathrm{u}}(1,5)$ & 16052990 & 120165 & & & & 1308958 & 20855 & 102648 & 37431 & 22993 & 4215 \\
\hline $\mathrm{B}_{2 \mathrm{u}}(4,7)$ & 17871153 & 297923 & 4406819 & $79921^{c}$ & $70971^{c}$ & 1360282 & 53515 & 132144 & 61684 & 25543 & 6301 \\
\hline $\mathrm{B}_{3 \mathrm{u}}(4,7)$ & 17871256 & 270331 & 4406819 & $76062^{c}$ & $63722^{c}$ & 1360282 & 49391 & 132169 & 62609 & 25543 & 6478 \\
\hline
\end{tabular}

${ }^{a}$ r-nos. denotes the number of one-electron excited configurations used as reference configurations in the configuration selection scheme in calculations B and C. Numbers on the left and right are the singlet and ionized reference numbers, respectively. ${ }^{b}$ The reference number is three. ${ }^{c}$ The reference number is five.

$2 \mathrm{~s}, 2 \mathrm{p}$ orbitals, and we added Rydberg s and $\mathrm{p}$ functions $\left(\alpha_{\mathrm{s}}=\right.$ $0.024, \alpha_{p}=0.022$ ) on all of the $\mathrm{N}$ atoms. The Rydberg exponents are the mean values of the four carbons and one nitrogen consisting of the pyrrole unit. The purpose of adding the Rydberg functions are two-fold: one is to calculate Rydberg excited states of FBP, and the second is to examine the valenceRydberg mixing, which can be eliminated only by adding proper Rydberg bases. The valence bases are Huzinaga's (63/5)/[3s2p] set $^{37}$ for carbon and nitrogen and a (4)/[1s] set ${ }^{38}$ for hydrogen. The total number of contracted GTOs is 246. The HartreeFock (HF) SCF orbitals used as the reference orbitals consist of 81 occupied and 165 unoccupied MOs. The SAC/SAC-CI calculations were carried out using $\mathrm{SAC} 85^{39}$ and its modified version, ${ }^{40}$ within the 57 higher occupied MOs and the 144 lower unoccupied MOs included in the active space. The total number of active orbitals was 201: all of the valence and Rydberg orbitals were included in the active space, and only the $1 \mathrm{~s}$ orbitals of $\mathrm{C}$ and $\mathrm{N}$ atoms were treated as frozen cores. All of the single excitations and selected double excitations within this active space constituted linked operators, and their products unlinked operators. The perturbative configuration selection procedure $^{14,41}$ was performed with the energy thresholds of 1 $\times 10^{-5}$ au $\left(\pi-\pi^{*}\right)$ and $2 \times 10^{-5}$ au (others) for the ground state, and $5 \times 10^{-7}$ au $\left(\pi-\pi^{*}\right)$ and $1 \times 10^{-6}$ au (others) for the excited states.

In calculation $B$, the basis set is double- $\zeta$ quality for the valence $2 s, 2 p$ orbitals, the same as in calculation $\mathrm{A}$, plus polarization d-functions $(\mathrm{C} ; \alpha=0.600 ; \mathrm{N}, \alpha=0.864)$ on all of the $\mathrm{C}$ and $\mathrm{N}$ atoms, but does not include the Rydberg functions. The total number of contracted GTOs is 374 . The HF SCF orbitals consist of 81 occupied and 293 unoccupied MOs. In the SAC/SAC-CI calculations, the 57 higher occupied MOs and the 289 lower unoccupied MOs, a total number of 346 MOs, were included in the active space: all of the valence orbitals were included in the active space, and only the $1 \mathrm{~s}$ orbitals of $\mathrm{C}$ and $\mathrm{N}$ atoms were treated as frozen cores. The configuration selection ${ }^{14,41}$ was performed with an energy threshold of $5 \times 10^{-6}$ au for the ground state, and with thresholds of $2 \times 10^{-7}$ and $7 \times 10^{-7}$ au for the excited and ionized states, respectively. We also performed SAC-CI calculations further including $\langle\mathrm{R}(1)|\mathrm{H}| \mathrm{R}(1) \mathrm{S}(1)\rangle$ and $\langle\mathrm{R}(2)|\mathrm{H}| \mathrm{R}-$ (1) $\mathrm{S}(1)\rangle$ unlinked terms to examine the effect of $\mathrm{S}(1)$ on the excitation energies, and this calculation is called calculation B2.
Note that $\mathrm{S}(1)$ is the SAC single-excitation operator and $\mathrm{R}(1)$ and $\mathrm{R}(2)$ are the SAC-CI single and double-excitation operators, respectively.

We compare the present results with the previous calculation, ${ }^{14}$ called here calculation $\mathrm{C}$. The basis set there was double- $\zeta$ quality only for $2 \mathrm{p}$ AOs of carbon and nitrogen and no Rydberg and polarization functions were added. The total number of contracted GTOs was 206. The HF SCF orbitals consisted of 81 occupied and 125 unoccupied MOs, and among these 42 higher occupied and 114 lower unoccupied MOs were included in the active space of the SAC/SAC-CI calculations. The total number of active orbitals was 156 , in comparison with 201 in calculation A and 346 in calculation B. The thresholds of the configuration selection were the same as in calculation A. We have performed two different calculations in which only the thresholds of the configuration selection were changed. One calculation $\mathrm{C} 2$ was performed with the energy thresholds of 1 $\times 10^{-5}$ au for the ground state and $5 \times 10^{-7}$ au for the excited states. The other calculation C3 was performed with energy thresholds of $6 \times 10^{-6}$ au for the ground state and $1 \times 10^{-7} \mathrm{au}$ for the excited states. The resultant dimensions for the $\mathrm{SAC} /$ SAC-CI calculations are shown in Tables 1 and 3.

\section{Electronic Structure of the Ground State}

We investigated the effects of Rydberg orbitals on the HF orbital energy by comparing calculations $\mathrm{A}$ and $\mathrm{C}^{14}$ shown in Table 2. Some important MOs are illustrated in Figure 2. In the case of pyrrole, ${ }^{32}$ the unoccupied valence $\pi$ orbitals have some diffuse character: the second moments perpendicular to the molecular plane for unoccupied $\pi_{4} *$ and $\pi_{5} *$ orbitals were 16.06 and $10.77 \mathrm{a}_{0}^{2}$, respectively, while those for occupied $\pi_{2}$ and $\pi_{3}$ are 2.28 and $2.51 \mathrm{a}_{0}^{2}$, respectively. ${ }^{32}$ This is why it is believed that Rydberg-valence mixing ${ }^{34}$ may occur in the $\pi^{*}$ orbitals of FBP and that the energy and oscillator strength of the allowed $\pi-\pi^{*}$ excited states may be affected by changes in the nature of the valence $\pi^{*}$ orbital. However, as shown in Table 2, the energy and the nature of the valence orbitals in calculation A are almost the same as those in calculation $\mathrm{C}$, except for the orbitals with Rydberg nature. The second moments perpendicular to the molecular plane for the $\pi$ orbitals, $56 \mathrm{MO}$ (next-HOMO), 57MO (HOMO), 58MO (LUMO), and 59MO (next-LUMO) are 4.829, 4.876, 5.086, and $5.120 \mathrm{a}_{0}^{2}$, 
TABLE 2: Hartree-Fock Orbital Energy and Character of Free-Base Porphin in Calculations A, B, and C

\begin{tabular}{|c|c|c|c|c|c|}
\hline \multirow{2}{*}{$\begin{array}{l}\mathrm{MO} \\
\text { no. }^{a}\end{array}$} & \multirow{2}{*}{$\begin{array}{l}\text { sym- } \\
\text { metry }\end{array}$} & \multicolumn{3}{|c|}{$\begin{array}{c}\text { orbital energy }(\mathrm{eV}) \\
\text { calculations }\end{array}$} & \multirow[b]{2}{*}{ character } \\
\hline & & A & B & $\mathrm{C}$ & \\
\hline \multicolumn{6}{|c|}{ Occupied Orbitals } \\
\hline 45 & $b_{3 u}$ & -14.414 & -13.149 & -14.484 & $\sigma$ \\
\hline 46 & $b_{1 g}$ & -14.325 & -12.885 & -14.355 & $\sigma$ \\
\hline 47 & $a_{u}$ & -12.147 & -12.061 & -12.216 & $\pi$ \\
\hline 48 & $\mathrm{~b}_{2 \mathrm{u}}$ & -11.386 & -11.574 & -11.415 & $\mathrm{n}$ \\
\hline 49 & $a_{g}$ & -11.257 & -11.476 & -11.263 & $\mathrm{n}$ \\
\hline 50 & $\mathrm{~b}_{1 \mathrm{u}}$ & -10.586 & -10.592 & -10.679 & $\pi$ \\
\hline 51 & $b_{2 g}$ & -10.541 & -10.555 & -10.632 & $\pi$ \\
\hline 52 & $b_{3 g}$ & -10.514 & -10.459 & -10.590 & $\pi$ \\
\hline 53 & $b_{2 g}$ & -10.252 & -10.187 & -10.321 & $\pi$ \\
\hline 54 & $b_{1 u}$ & -9.249 & -9.250 & -9.327 & $\pi$ \\
\hline 55 & $b_{3 g}$ & -9.067 & -9.082 & -9.122 & $\pi$ \\
\hline 56 & $\mathrm{~b}_{1 \mathrm{u}}$ & -6.631 & -6.827 & -6.686 & $\pi$ \\
\hline 57 & $a_{u}$ & -6.485 & -6.200 & -6.521 & $\pi$ \\
\hline \multicolumn{6}{|c|}{ Unoccupied Orbitals } \\
\hline 58 & $b_{2 g}$ & -0.031 & 0.065 & -0.069 & $\pi$ \\
\hline 59 & $b_{3 g}$ & 0.164 & 0.176 & 0.141 & $\pi$ \\
\hline$(60)$ & $b_{3 u}$ & 1.589 & & & $\sigma, \operatorname{Ryd}\left(3 \mathrm{~s}, 3 \mathrm{p}_{x}\right)$ \\
\hline (61) & $b_{2 u}$ & 1.675 & & & $\sigma, \operatorname{Ryd}\left(3 \mathrm{~s}, 3 \mathrm{p}_{v}\right)$ \\
\hline (62) & $a_{g}$ & 1.744 & & & $\sigma, \operatorname{Ryd}\left(3 \mathrm{~s}, 3 \mathrm{p}_{x}, 3 \mathrm{p}_{y}\right)$ \\
\hline (63) & $\mathrm{ag}_{\mathrm{g}}$ & 2.001 & & & $\sigma, \operatorname{Ryd}\left(3 \mathrm{~s}, 3 \mathrm{p}_{y}\right)$ \\
\hline (64) & $\mathrm{b}_{1 \mathrm{u}}$ & 2.185 & & & $\pi, \operatorname{Ryd}\left(3 \mathrm{p}_{z}\right)$ \\
\hline (65) & $b_{2 g}$ & 2.553 & & & $\pi, \operatorname{Ryd}\left(3 \mathrm{p}_{z}\right)$ \\
\hline (66) & $b_{1 g}$ & 2.621 & & & $\sigma, \operatorname{Ryd}\left(3 \mathrm{p}_{y}\right)$ \\
\hline (67) & $b_{3 g}$ & 2.659 & & & $\pi, \operatorname{Ryd}\left(3 \mathrm{p}_{z}\right)$ \\
\hline $60(68)$ & $a_{u}$ & 2.844 & 3.014 & 2.842 & $\pi$ \\
\hline (69) & $b_{3 u}$ & 2.957 & & & $\sigma, \operatorname{Ryd}\left(3 \mathrm{~s}, 3 \mathrm{p}_{x}\right)$ \\
\hline (70) & $\mathrm{b}_{2 \mathrm{u}}$ & 2.982 & & & $\sigma, \operatorname{Ryd}\left(3 \mathrm{~s}, 3 \mathrm{p}_{y}\right)$ \\
\hline (71) & $b_{1 u}$ & 3.037 & & & $\pi, \operatorname{Ryd}\left(3 \mathrm{p}_{z}\right)$ \\
\hline (72) & $b_{1 g}$ & 3.146 & & & $\sigma, \operatorname{Ryd}\left(3 \mathrm{p}_{x}, 3 \mathrm{p}_{y}\right)$ \\
\hline (73) & $a_{g}$ & 3.588 & & & $\sigma, \operatorname{Ryd}\left(3 \mathrm{p}_{x}, 3 \mathrm{p}_{y}\right)$ \\
\hline (74) & $b_{3 u}$ & 3.904 & & & $\sigma, \operatorname{Ryd}\left(3 \mathrm{p}_{x}\right)$ \\
\hline (75) & $\mathrm{b}_{2 \mathrm{u}}$ & 4.097 & & & $\sigma, \operatorname{Ryd}\left(3 \mathrm{p}_{y}\right)$ \\
\hline (76) & $a_{g}$ & 4.605 & & & $\sigma, \operatorname{Ryd}\left(3 \mathrm{p}_{x}, 3 \mathrm{p}_{y}\right)$ \\
\hline $61(77)$ & $\mathrm{b}_{1 \mathrm{u}}$ & 5.116 & 5.002 & 5.002 & $\pi$ \\
\hline 62 & $a_{g}$ & & 5.485 & 6.917 & $\sigma$ \\
\hline $63(79)$ & $b_{3 g}$ & 5.719 & 5.756 & 5.702 & $\pi$ \\
\hline $64(78)$ & $b_{2 g}$ & 5.712 & 5.785 & 5.698 & $\pi$ \\
\hline 65 & $b_{3 u}$ & & 5.809 & 6.852 & $\sigma$ \\
\hline 66 & $\mathrm{~b}_{2 \mathrm{u}}$ & & 5.915 & 7.437 & $\sigma$ \\
\hline $67(80)$ & $a_{u}$ & 6.183 & 6.306 & 6.177 & $\pi$ \\
\hline
\end{tabular}

${ }^{a}$ The numbering of the Hartree-Fock orbitals in calculation A including Rydberg orbitals is shown in the brackets.

TABLE 3: Excited ${ }^{1} B_{3 U}$ States of Free-Base Porphin by the SAC-CI Method Due to Different Configuration Selection Thresholds in Calculation C

\begin{tabular}{ccrrrrrr}
\hline & \multicolumn{2}{c}{ dimensions } & & \multicolumn{2}{c}{ excitation energy $(\mathrm{eV})$} & \\
\cline { 2 - 3 } calculation & ground & excited & & $1^{1} \mathrm{~B}_{3 \mathrm{u}}$ & $2^{1} \mathrm{~B}_{3 \mathrm{u}}$ & $\Delta(\mathrm{eV})^{a}$ \\
\hline $\mathrm{C}$ & 10879 & 49391 & & 1.75 & 3.56 & 1.81 \\
$\mathrm{C} 2$ & 31404 & 64300 & & 1.96 & 3.77 & 1.81 \\
$\mathrm{C} 3$ & 60988 & 149638 & & 1.94 & 3.78 & 1.84 \\
exptl $^{b}$ & & & & 1.98 & 3.33 & 1.35
\end{tabular}

${ }^{a} \Delta$ is the difference in energy between the $2^{1} \mathrm{~B}_{3 \mathrm{u}}$ and $1^{1} \mathrm{~B}_{3 \mathrm{u}}$ states. ${ }^{b}$ Reference 27.

respectively, while those of the Rydberg 64, 65 and 67MOs are $79.868,82.182$ and $82.361 \mathrm{a}_{0}^{2}$, respectively. Thus, the Rydberg-valence mixing is not observed in the $\pi^{*}$ orbitals of FBP.

The HF orbital energy is significantly affected by the polarization functions as seen from calculations $\mathrm{B}$ and $\mathrm{C}$ shown in Table 2 . The porphyrin $\sigma$-type orbitals vary greatly, while the $\pi$ and $n$ orbitals change only a little. This indicates that the polarization functions have a large reorganization effect on (a)
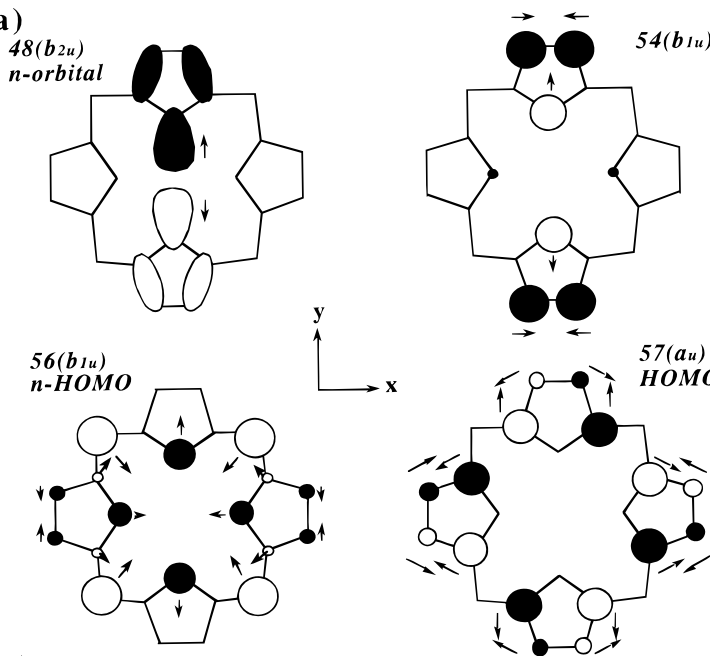

(b)
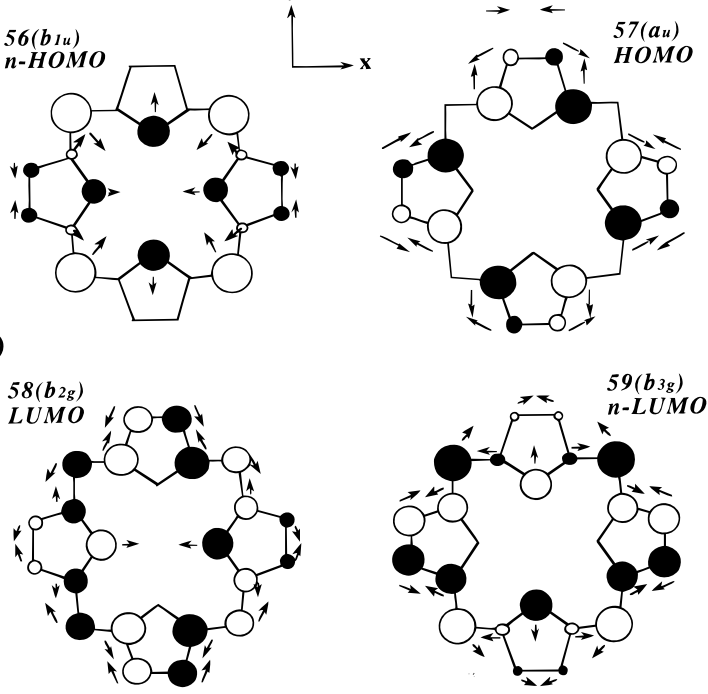

Figure 2. Orbitals for (a) the occupied MOs $(48,54,56,57)$ and (b) the unoccupied MOs $(58,59)$. The arrows show the polarization of $\pi$ orbital due to the addition of polarization d-function.

the $\sigma$ orbitals. The occupied and unoccupied $\sigma$-orbital energies are unstabilized and stabilized by about $1.2 \mathrm{eV}$, respectively. On the other hand, the $\pi$-orbital energies are slightly unstabilized by about $0.1 \mathrm{eV}$, except for 56MO (next-HOMO), which is stabilized by about $0.2 \mathrm{eV}$, and 57MO (HOMO), which is unstabilized by about $0.3 \mathrm{eV}$. The mechanism of the mixing of the polarization functions in the HF orbitals was studied earlier by Nakatsuji and Musher. ${ }^{43}$ In the occupied orbitals, the effect of the polarization function is generally to unstabilize the orbital energy rather than stabilize, ${ }^{43}$ though the total energy is of course stabilized by the addition of polarization functions.

The effects of the polarization d-functions on the $\pi$ orbitals of FBP are illustrated in Figure 2. In the HOMO, the p $\pi$ AOs are polarized toward the $\mathrm{p} \pi$ AOs of the adjacent atoms as shown by the arrows, by the addition of the polarization d-functions, so that neighboring $\pi$-electron repulsion increases and the MO energy is destabilized. The effect of the polarization d-functions on the other $\pi$ MOs are exhibited similarly in Figure 2. The polarization functions tend to polarize the $\mathrm{p} \pi$ functions in such a way to increase the positive (bonding) overlap between the adjacent AOs. On the other hand, in the next-HOMO, the neighboring atoms share little $\pi$-electron density, and therefore, the energy of this MO is only slightly affected by the polarization d-functions. Thus, the energy of the next-HOMO is slightly stabilized rather than unstabilized. ${ }^{43}$ In the $n$ orbital $\left(48\left(b_{2 u}\right)\right)$ the electron density of the lone-pair opposing nitrogens is reduced by the polarization function, so that this orbital is stabilized by about $0.2 \mathrm{eV}$.

The SAC correlation energies in calculations A and B for the ground state are 10.2 and $19.7 \mathrm{eV}$, respectively, in comparison with those of calculation $\mathrm{C}$ and the MRSDCI calculation, ${ }^{12}$ which give correlation energies of $10.0^{14}$ and 12.6 $\mathrm{eV},{ }^{12}$ respectively. 
TABLE 4: Excited States of Free-Base Porphin Calculated by SAC-CI Calculation A ${ }^{e}$

\begin{tabular}{|c|c|c|c|c|c|c|c|c|c|c|c|}
\hline \multicolumn{11}{|c|}{ SAC-CI } & \multirow{4}{*}{$\begin{array}{c}\operatorname{exptl}^{a} \\
\text { excitation } \\
\text { energy } \\
(\mathrm{eV})\end{array}$} \\
\hline \multicolumn{5}{|c|}{ calculation A } & & & & & & \multirow{3}{*}{ 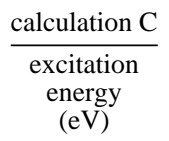 } & \\
\hline & & & excitation $^{d}$ & & & \multicolumn{4}{|c|}{ second moment ${ }^{b}$} & & \\
\hline state & & nature & & $\begin{array}{l}\text { polario- } \\
\text { zation }\end{array}$ & $\begin{array}{l}\text { Oscillator } \\
\text { strength }\end{array}$ & $\left\langle x^{2}\right\rangle$ & $\left\langle y^{2}\right\rangle$ & $\left\langle z^{2}\right\rangle$ & $\left\langle R^{2}\right\rangle$ & & \\
\hline $1^{1} \mathrm{~B}_{3 \mathrm{u}}$ & $0.72(56-58)-0.60(57-59)$ & $\pi-\pi^{*}$ & $1.70(-0.05)$ & $x$ & $1.91 \times 10^{-3}$ & -81.449 & -91.754 & -114.182 & -287.386 & 1.75 & $1.98 \mathrm{Q}_{x}$ \\
\hline $1^{1} \mathrm{~B}_{2 \mathrm{u}}$ & $-0.69(57-58)-0.66(56-59)$ & $\pi-\pi^{*}$ & $2.19(-0.04)$ & $y$ & $5.19 \times 10^{-3}$ & -79.664 & -93.265 & -114.182 & -287.225 & 2.23 & $2.42 \mathrm{Q}_{y}$ \\
\hline $2^{1} \mathrm{~B}_{3 \mathrm{u}}$ & $\begin{array}{l}-0.65(57-59)+0.51(54-58) \\
-0.44(56-58)\end{array}$ & $\pi-\pi^{*}$ & $3.43(-0.13)$ & $x$ & 1.10 & -86.267 & -87.470 & -114.370 & -288.107 & 3.56 & $3.33 \mathrm{~B}$ \\
\hline $2^{1} \mathrm{~B}_{2 \mathrm{u}}$ & $\begin{array}{l}0.64(56-59)-0.63(57-58) \\
-0.28(54-59)\end{array}$ & $\pi-\pi^{*}$ & $3.62(-0.13)$ & $y$ & 1.87 & -84.065 & -91.064 & -114.449 & -289.578 & 3.75 & $3.65 \mathrm{~N}$ \\
\hline $3^{1} \mathrm{~B}_{3 \mathrm{u}}$ & $\begin{array}{l}0.76(54-58)+0.40(57-59) \\
-0.32(56-58)\end{array}$ & $\pi-\pi^{*}$ & $4.08(-0.16)$ & $x$ & 1.09 & -86.469 & -80.783 & -114.449 & -289.578 & 4.24 & $4.25 \mathrm{~L}$ \\
\hline $3{ }^{1} \mathrm{~B}_{2 \mathrm{u}}$ & $0.89(54-59)$ & $\pi-\pi^{*}$ & $4.36(-0.16)$ & $y$ & 0.437 & -96.419 & -70.339 & -114.477 & -281.235 & 4.52 & $4.67 \mathrm{~L}$ \\
\hline $4^{1} \mathrm{~B}_{2 \mathrm{u}}$ & $0.89(50-59)$ & $\pi-\pi^{*}$ & $5.15(-0.16)$ & $y$ & 0.290 & -66.983 & -97.943 & -114.482 & -279.408 & 5.31 & M \\
\hline $4^{1} \mathrm{~B}_{3 \mathrm{u}}$ & $-0.91(50-58)$ & $\pi-\pi^{*}$ & $5.25(-0.20)$ & $x$ & 0.412 & -56.633 & -108.795 & -114.492 & -279.919 & 5.45 & $5.50 \mathrm{M}$ \\
\hline $5^{1} \mathrm{~B}_{3 \mathrm{u}}$ & $0.96(56-65)$ & Ryd & 5.51 & $x$ & $9.21 \times 10^{-3}$ & -96.970 & -81.145 & -151.137 & -329.251 & & \\
\hline $5^{1} B_{2 u}$ & $0.96(56-67)$ & Ryd & 5.55 & $y$ & $3.82 \times 10^{-3}$ & -68.008 & -110.211 & -151.272 & -329.490 & & \\
\hline $6^{1} \mathrm{~B}_{2 \mathrm{u}}$ & $-0.96(57-65)$ & Ryd & 5.62 & $y$ & $1.85 \times 10^{-3}$ & -96.071 & -78.250 & -151.090 & -325.672 & & \\
\hline $6^{1} \mathrm{~B}_{3 \mathrm{u}}$ & $0.97(57-67)$ & Ryd & 5.79 & $x$ & $2.07 \times 10^{-3}$ & -67.543 & -106.778 & -151.351 & -325.672 & & \\
\hline $7^{1} \mathrm{~B}_{3 \mathrm{u}}$ & $0.87(55-68)$ & $\pi-\pi^{*}$ & 6.56 & $x$ & 0.297 & -95.231 & -84.745 & -115.259 & -295.275 & & \\
\hline $7^{1} B_{2 u}$ & $-0.88(47-58)$ & $\pi-\pi^{*}$ & 6.71 & $y$ & $2.66 \times 10^{-2}$ & -75.162 & -97.782 & -114.512 & -287.456 & & \\
\hline $8^{1} \mathrm{~B}_{2 \mathrm{u}}$ & $0.68(57-65)-0.59(55-64)$ & Ryd & 6.75 & $y$ & $1.54 \times 10^{-2}$ & -89.911 & -77.745 & -151.299 & -318.955 & & \\
\hline $8^{1} \mathrm{~B}_{3 \mathrm{u}}$ & $\begin{array}{l}-0.75(57-67)-0.42(56-78) \\
+0.33(50-58)\end{array}$ & $\pi-\pi^{*}$ & 6.82 & $x$ & $2.01 \times 10^{-4}$ & -81.181 & -92.985 & -114.438 & -288.605 & & \\
\hline $9^{1} \mathrm{~B}_{2 \mathrm{u}}$ & $0.90(56-79)$ & $\pi-\pi^{*}$ & 6.93 & $y$ & $4.00 \times 10^{-2}$ & -100.377 & -83.570 & -115.280 & -299.277 & & \\
\hline $9^{1} \mathrm{~B}_{3 \mathrm{u}}$ & $0.80(56-78)+0.42(47-59)$ & $\pi-\pi^{*}$ & 7.16 & $x$ & $1.48 \times 10^{-3}$ & -75.765 & -106.615 & -114.821 & -297.201 & & \\
\hline $10^{1} \mathrm{~B}_{2 \mathrm{u}}$ & $0.90(57-78)$ & $\pi-\pi^{*}$ & 7.21 & $y$ & $9.05 \times 10^{-2}$ & -76.304 & -102.332 & -114.929 & -293.565 & & \\
\hline $10^{1} \mathrm{~B}_{3 \mathrm{u}}$ & $-0.84(57-79)-0.40(50-58)$ & $\pi-\pi^{*}$ & 7.42 & $x$ & 0.143 & -92.987 & -85.675 & -114.868 & -293.530 & & \\
\hline
\end{tabular}

${ }^{a}$ Reference 27. ${ }^{b}$ Sums of second moments of electron and nuclear charges. ${ }^{c}$ Numbering of the orbitals is given in Table 2 and Figure $2 .{ }^{d}$ Values in parentheses show the difference from the result of calculation C. ${ }^{e}$ Only the symmetry-allowed states ${ }^{1} \mathrm{~B}_{2 u}$ and ${ }^{1} \mathrm{~B}_{3 u}$ were calculated

\section{Excited States}

(a) Effect of Selection. We first performed large-dimension calculations $\mathrm{C} 2$ and $\mathrm{C} 3$ using the same basis sets as in calculation C. ${ }^{14}$ These calculations were carried out only for $\mathrm{B}_{3 \mathrm{u}}$ states with $x$ polarization, and the results are shown in Table 3. We monitored the energy difference between $1^{1} B_{3 u}\left(Q_{x}\right.$ band $)$ and $2^{1} \mathrm{~B}_{3 \mathrm{u}}(\mathrm{B}$ band $)$, since the energies of these states were underand overestimated, respectively, in calculation $\mathrm{C}$. The excitation energy for the $1^{1} \mathrm{~B}_{3 \mathrm{u}}$ state was certainly improved by about 0.2 $\mathrm{eV}$, but the $2^{1} \mathrm{~B}_{3 \mathrm{u}}$ state is further overestimated by the same amount, the difference energy $\Delta$ being almost the same.

(b) Rydberg Excited States and the Effect of Rydberg Basis Set. We investigated the Rydberg excited states and the effect of Rydberg functions on the excitation energies of FBP. Table 4 gives detailed information about the excited states due to calculation A. We found that the Rydberg states appeared at 5.5-5.8 eV with small intensities, and therefore the assignment of the experimental peaks of FBP was the same as those with calculation C. ${ }^{14}$ The Rydberg orbitals shifts the $\mathrm{Q}$ bands by $0.05 \mathrm{eV}$ to lower energy and other valence excited states (B-M bands) by $0.13-0.2 \mathrm{eV}$ again to lower side: the Rydberg orbitals stabilize more the excited state than the ground state, as expected, and the effect increases as energy level of the excited state increases, again as expected. However, generally speaking, the Rydberg basis has only a secondary effect on the calculations of the valence excited states of FBP: the valenceRydberg mixing is small as expected from the HF MOs.

The Rydberg states are distinguished from the valence states using the second moment: in Table 4, the second moments $\left\langle z^{2}\right\rangle$ of the Rydberg states are about $150 \mathrm{a}_{0}^{2}$, while those of the typical valence states are $114 \mathrm{a}_{0}^{2}$. The oscillator strengths of these Rydberg states are $10^{-2}-10^{-3}$, which are much smaller than those of the valence states except for the $\mathrm{Q}_{x}\left(1^{1} \mathrm{~B}_{3 \mathrm{u}}\right)$ and $\mathrm{Q}_{y}$ $\left(1^{1} B_{2 u}\right)$ states. The $8^{1} B_{2 u}$ state has an oscillator strength of 1.54 $\times 10^{-2}$. Thus, although the $\mathrm{M}$ band contains valence $\pi-\pi^{*}$ states $\left(4^{1} \mathrm{~B}_{2 u}\right.$ and $\left.4^{1} \mathrm{~B}_{3 u}\right)$ and Rydberg states $\left(5^{1} \mathrm{~B}_{3 \mathrm{u}}, 5^{1} \mathrm{~B}_{2 \mathrm{u}}, 6^{1} \mathrm{~B}_{2 \mathrm{u}}\right.$, and $\left.6^{1} \mathrm{~B}_{3 \mathrm{u}}\right)$, the Rydberg states have almost no effect on the shape of the $M$ band. Though several valence and Rydberg states were calculated at the energy region higher than that observed, i.e., $5.5 \mathrm{eV}$, only the $7^{1} \mathrm{~B}_{3 \mathrm{u}}$ and $10^{1} \mathrm{~B}_{3 \mathrm{u}}$ states have large oscillator strengths, 0.297 and 0.143 , respectively, so that the existence of new bands is indicated at 6.6 and $7.4 \mathrm{eV}$.

(c) Effect of Polarization Function. In calculation B, we calculated the excited states of FBP over a wide energy range from visible light to $\mathrm{UV}$ up to $5.8 \mathrm{eV}$. The polarization of electron cloud was poorly described in calculation $\mathrm{C}$, since it used only a 2 p double- $\zeta$ basis set. On the other hand, calculation B uses a valence double- $\zeta$ basis set plus polarization d-functions, so that such polarization of electron cloud should be described much better, and this calculation may be expected to improve the excitation energies compared with calculation $\mathrm{C}$.

In Figure 3, the SAC-CI B and C theoretical spectra are compared with the experimental spectrum in the vapor phase as measured by Edwards and Dolphin, ${ }^{25}$ and Table 5 gives more detailed information on the excited states. The assignments of the main peaks having large oscillator strengths are the same as those due to calculation $C$. In addition, the energies and oscillator strengths of the excited states are similar to those with calculation C. However, the effects of the polarization dfunctions are not monotonic to the excitation energies, unlike the effect of the Rydberg functions: some levels are shifted up but some levels are shifted down. The width is -0.22 to +0.19 $\mathrm{eV}$ as seen from the values in parentheses in Table 5. As seen from Figure 3, these shifts are generally small, so that the polarization function does not have a large effect on the excited states and that calculation $\mathrm{C}$ was almost valid for the excited states, though the dimensions calculated were small.

We see from Table 5 that the $\pi-\pi^{*}$ excited states are generally stabilized by the addition of the polarization functions, 
TABLE 5: Excited States of Free-Base Porphin Calculated by SAC-CI Calculations B and B2

\begin{tabular}{|c|c|c|c|c|c|c|c|c|c|c|}
\hline \multirow[b]{3}{*}{ state $^{a}$} & \multicolumn{9}{|c|}{ SAC-CI } & \multirow{3}{*}{$\begin{array}{c}\text { exptl }^{c} \\
\text { excitation } \\
\text { energy } \\
(\mathrm{eV})\end{array}$} \\
\hline & \multicolumn{5}{|c|}{ calculation B } & \multicolumn{3}{|c|}{ calculation $\mathrm{C}$} & \multirow{2}{*}{ 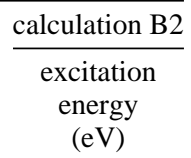 } & \\
\hline & $\begin{array}{l}\text { main configurations }^{b} \\
\qquad(|C|>0.25)\end{array}$ & nature & $\begin{array}{l}\text { excitation }^{d} \\
\text { energy } \\
(\mathrm{eV})\end{array}$ & $\begin{array}{l}\text { polari- } \\
\text { zation }\end{array}$ & $\begin{array}{l}\text { oscillator } \\
\text { strength }\end{array}$ & $\begin{array}{l}\text { polari- } \\
\text { zation }\end{array}$ & $\begin{array}{l}\text { oscillator } \\
\text { strength }\end{array}$ & $\begin{array}{c}\text { excitation } \\
\text { energy } \\
(\mathrm{eV})\end{array}$ & & \\
\hline $\mathbf{1}^{1} \mathbf{B}_{3 u}$ & $0.71(56-58)-0.66(57-59)$ & $\pi-\pi^{*}$ & $1.77(0.02)$ & $x$ & $2.80 \times 10^{-3}$ & $x$ & $1.13 \times 10^{-3}$ & 1.75 & 1.71 & $1.98 \mathrm{Q}_{x}$ \\
\hline $\mathbf{1}^{1} \mathbf{B}_{2 u}$ & $-0.71(57-58)-0.66(56-59)$ & $\pi-\pi^{*}$ & $2.01(-0.22)$ & $y$ & $1.32 \times 10^{-2}$ & $y$ & $5.66 \times 10^{-3}$ & 2.23 & 1.96 & $2.42 \mathrm{Q}_{y}$ \\
\hline $1^{1} \mathrm{~B}_{1 \mathrm{~g}}$ & $-0.94(55-58)$ & $\pi-\pi^{*}$ & $3.45(-0.10)$ & & 0.0 & & 0.0 & 3.55 & & \\
\hline $2^{1} \mathbf{B}_{3 \mathbf{u}}$ & $\begin{array}{l}0.61(54-58)-0.58(57-59) \\
-0.44(56-58)\end{array}$ & $\pi-\pi^{*}$ & $3.47(-0.09)$ & $x$ & 0.772 & $x$ & 1.03 & 3.56 & 3.44 & $3.33 \mathrm{~B}$ \\
\hline $2^{1} \mathbf{B}_{2 u}$ & $\begin{array}{l}0.67(56-59)-0.63(57-58) \\
-0.28(54-59)\end{array}$ & $\pi-\pi^{*}$ & $3.73(-0.02)$ & $y$ & 1.62 & $y$ & 1.73 & 3.75 & 3.71 & $3.65 \mathrm{~N}$ \\
\hline $3^{1} \mathbf{B}_{3 \mathbf{u}}$ & $\begin{array}{l}-0.71(54-58)-0.47(56-58) \\
-0.40(57-59)\end{array}$ & $\pi-\pi^{*}$ & $4.20(-0.04)$ & $x$ & 1.32 & $x$ & 0.976 & 4.24 & 4.16 & $4.25 \mathrm{~L}$ \\
\hline $1^{1} \mathrm{~B}_{2 \mathrm{~g}}$ & $0.95(49-58)$ & $\mathrm{n}-\pi^{*}$ & $4.22(0.17)$ & & 0.0 & & 0.0 & 4.05 & & \\
\hline $2^{1} \mathrm{~A}_{\mathrm{g}}$ & $-0.94(55-59)$ & $\pi-\pi^{*}$ & $4.24(-0.05)$ & & 0.0 & & & 4.29 & & \\
\hline $1^{1} \mathrm{~A}_{\mathrm{u}}$ & $-0.94(48-58)$ & $\mathrm{n}-\pi^{*}$ & $4.32(0.14)$ & & 0.0 & & 0.0 & 4.18 & & \\
\hline $3^{1} \mathbf{B}_{2 u}$ & $-0.89(54-59)+0.27(50-59)$ & $\pi-\pi^{*}$ & $4.38(-0.14)$ & $y$ & 0.339 & $y$ & 0.350 & 4.52 & 4.35 & $4.67 \mathrm{~L}$ \\
\hline $1^{1} \mathrm{~B}_{3 \mathrm{~g}}$ & $0.95(49-59)$ & $\mathrm{n}-\pi^{*}$ & $4.51(0.19)$ & & 0.0 & & 0.0 & 4.37 & & \\
\hline $\mathbf{1}^{1} \mathbf{B}_{1 \mathrm{u}}$ & $0.95(48-59)$ & $\mathrm{n}-\pi^{*}$ & $4.63(0.12)$ & $z$ & $3.02 \times 10^{-3}$ & $z$ & $5.30 \times 10^{-3}$ & 4.51 & & $\mathrm{~L}$ \\
\hline $3^{1} \mathrm{Ag}_{\mathrm{g}}$ & $0.94(57-60)$ & $\pi-\pi^{*}$ & $4.74(0.0)$ & & 0.0 & & 0.0 & 4.74 & & \\
\hline $4^{1} B_{2 u}$ & $-0.89(50-59)$ & $\pi-\pi^{*}$ & $5.15(-0.16)$ & $y$ & 0.319 & $y$ & 0.280 & 5.31 & 5.13 & M \\
\hline $2^{1} B_{1 g}$ & $\begin{array}{l}-0.72(53-59)+0.59(52-58) \\
+0.25(51-59)\end{array}$ & $\pi-\pi^{*}$ & 5.22 & & 0.0 & & 0.0 & & & \\
\hline $4^{1} B_{3 u}$ & $-0.94(50-58)$ & $\pi-\pi^{*}$ & $5.44(-0.01)$ & $x$ & 0.295 & $x$ & 0.351 & 5.45 & 5.39 & $5.50 \mathrm{M}$ \\
\hline $3^{1} \mathrm{~B}_{1 \mathrm{~g}}$ & $-0.84(51-59)+0.43(52-58)$ & $\pi-\pi^{*}$ & 5.80 & & 0.0 & & 0.0 & & & \\
\hline
\end{tabular}

${ }^{a}$ Boldface letters show optically allowed states. ${ }^{b}$ Numbering of the orbitals is given in Table 2 and Figure 2. ${ }^{c}$ Reference 27. ${ }^{d}$ Values in parentheses show the difference from the result of calculation $\mathrm{C}$.

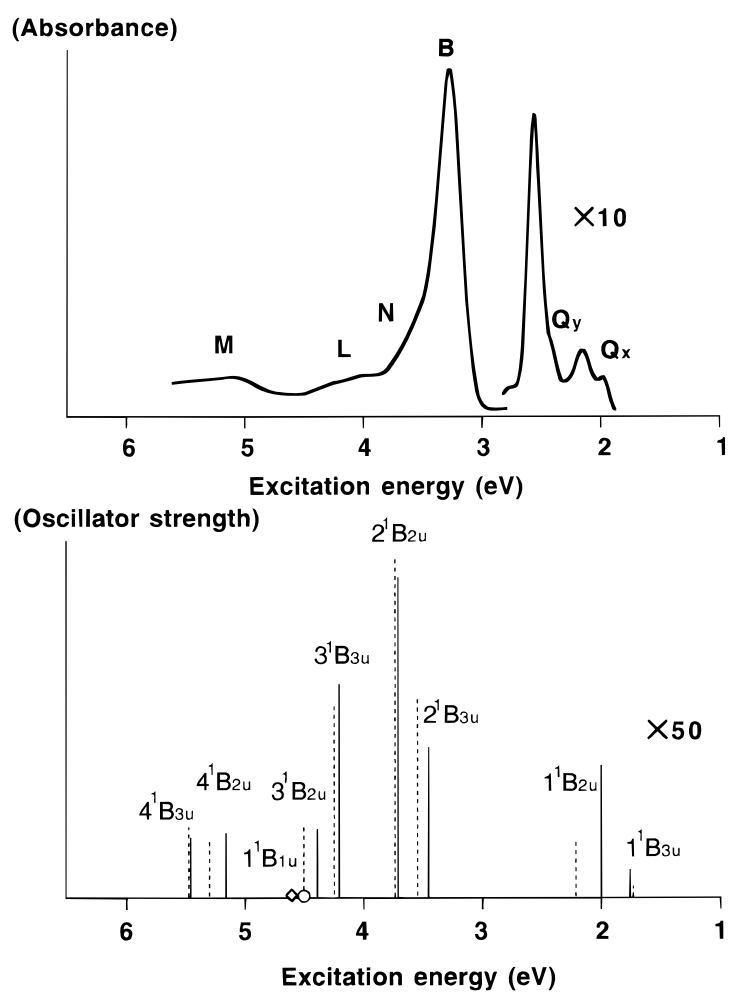

Figure 3. Electronic spectra of free-base porphin: (a) gas-phase experimental spectrum from Edwards and Dolphin and (b) SAC-CI theoretical spectra. The solid and dotted lines are the results of calculations B and C, respectively. The $1^{1} \mathrm{~B}_{1 \mathrm{u}}$ states of calculations B and $C$ are indicated by the open diamond $(\diamond)$ and circle $(O)$, respectively.

except for the $\mathrm{Q}_{x}$ state, while the $\mathrm{n}-\pi^{*}$ states are destabilized. The polarization d-functions have larger stabilization effects on the excited states than on the ground state. This is the same as the Rydberg functions studied above. On the other hand, the energies of the $n-\pi^{*}$ states are $0.12-0.19 \mathrm{eV}$ higher than those with calculation C. This is explained by the fact that the HF $n$ orbitals are much stabilized by the polarization d-functions (Table 2).

In Figure 3, the $1^{1} \mathrm{~B}_{2 \mathrm{u}}\left(\mathrm{Q}_{y}\right), 2^{1} \mathrm{~B}_{3 \mathrm{u}}(\mathrm{B}), 3^{1} \mathrm{~B}_{2 \mathrm{u}}(\mathrm{L})$, and $4^{1} \mathrm{~B}_{2 \mathrm{u}}$ (M) states are more largely stabilized than the other states by the addition of the polarization d-functions. In case of $1^{1} \mathrm{~B}_{2 \mathrm{u}}$ state, it is expected that the excitation energy is affected by the energy change of the Gouterman 4 orbitals, ${ }^{1}$ because the $\mathrm{Q}$ bands can be described well by the 4-orbital model. ${ }^{1,14}$ The orbital energy difference for the excitation from MO 57 to 58, which is one of the main configurations, decreases by $0.317 \mathrm{eV}$ compared with that of calculation $\mathrm{C}$, whereas the other counterpart, the excitation from MO 56 to 59 , increases by 0.176 $\mathrm{eV}$. Since the contributions of the both configurations to the excitation energy are almost equal (Table 5), 0.71 and 0.66, respectively, the $1^{1} \mathrm{~B}_{2 \mathrm{u}}$ state energy decreases. On the other hand, for the $1^{1} \mathrm{~B}_{3 \mathrm{u}}\left(\mathrm{Q}_{x}\right)$ state the orbital energy differences between the MOs 56 and 58 and MOs 57 and 59 increase and decrease by 0.275 and $0.286 \mathrm{eV}$, respectively, compared with those of calculation $\mathrm{C}$, and the coefficients of the former and latter configurations are comparable, 0.71 and 0.66 , so that the effects are almost canceled out. Thus, the $1^{1} \mathrm{~B}_{3 \mathrm{u}}$ state energy scarcely changes.

The $2^{1} \mathrm{~B}_{3 \mathrm{u}}$ (B) state has three main configurations. Two of them are the same as the $1^{1} \mathrm{~B}_{3 \mathrm{u}}$ state; and the other is the configuration from MO 54 to 58 , which is dominant in calculation B: the Gouterman 4-orbital model does not hold for the B state. As shown in the previous paper, ${ }^{14}$ the B state is a typical ionic state, so that reorganization of electron cloud following the excitation is important and therefore the polarization d-functions work to stabilize the $\mathrm{B}$ state.

The $3^{1} \mathrm{~B}_{2 \mathrm{u}}(\mathrm{L})$ and $4^{1} \mathrm{~B}_{2 \mathrm{u}}(\mathrm{M})$ states, both being $y$-polarized, have the main configurations from MO 54 to 59 and 50 to 59, respectively, and they are stabilized by 0.14 and $0.16 \mathrm{eV}$, respectively. On the other hand, the counterpart $x$-polarized states $3^{1} \mathrm{~B}_{3 \mathrm{u}}(\mathrm{L})$ and $4^{1} \mathrm{~B}_{3 \mathrm{u}}(\mathrm{M})$ states have main configurations from MO 54 to 58 and 50 to 58 , respectively, and they are 


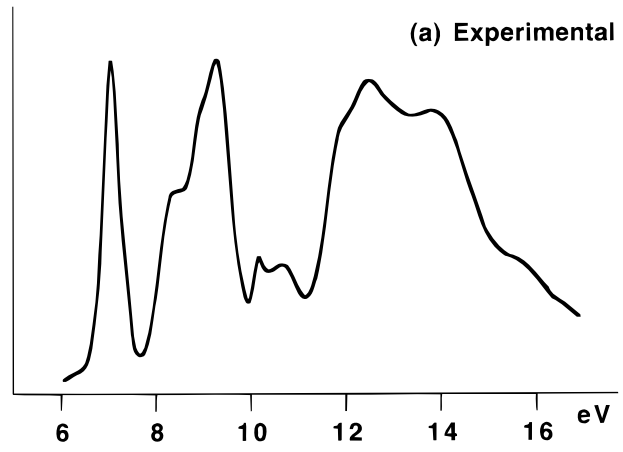

$S A C-C I$

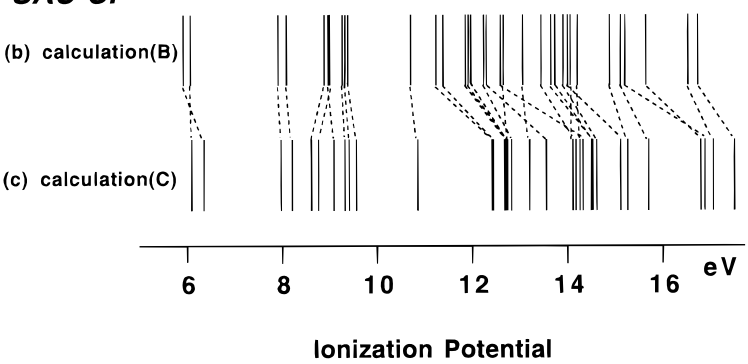

Figure 4. Ionization spectra of free-base porphin. (a) Photoelectron spectrum. (b) and (c) are the ionization potentials of SAC-CI calculations $\mathrm{B}$ and $\mathrm{C}$, respectively. The dotted lines show correspondence between the ionization potentials of calculations $\mathrm{B}$ and $\mathrm{C}$.

scarcely stabilized (0.04 and $0.01 \mathrm{eV}$, respectively). This is understood from the change in the orbital energy shown in Table 2. All the MOs involved are destabilized by the addition of the polarization d-functions except for the MO 59, and therefore, the excitations to MO 59 are stabilized relatively more largely than the excitations to MO 58.

We also performed calculations for the ${ }^{1} B_{2 u}$ and ${ }^{1} B_{3 u}$ states, adding the $\langle\mathrm{R}(1)|\mathrm{H}| \mathrm{R}(1) \mathrm{S}(1)\rangle$ and $\langle\mathrm{R}(2)|\mathrm{H}| \mathrm{R}(1) \mathrm{S}(1)\rangle$ terms in the unlinked term of SAC-CI in calculation B2. However, the effects of these unlinked terms on the excitation energies were small, as shown in Table 5.

\section{Ionized States}

The photoelectron spectrum of FBP was observed in the vapor phase by Dupuis et al. ${ }^{27}$ and is shown in Figure 4. SAC-CI calculations $\mathrm{B}$ and $\mathrm{C}$ are compared with the experimental spectrum in Figure 4 and Table 6 . The dimensions of calculation B are about 10 times larger than those of calculation $\mathrm{C}$, as shown in Table 1. The polarization function has a large reorganization effect for the $\mathrm{n}$ and $\sigma$ states, whereas this effect is small for the $\pi$ states. In particular, the energies of the $\sigma$ states are lowered by as large as $1 \mathrm{eV}$ by the addition of the polarization functions. This result is similar to that in our previous investigation of benzene. ${ }^{33}$

The first and second ionized states are exchanged from those of calculation C. ${ }^{14}$ This is caused by the fact that the HOMO energy is unstabilized and the next-HOMO energy is slightly stabilized due to the effect of the polarization d-function, as described in section III. Actually, only the energy of the $1^{2} \mathrm{~A}_{\mathrm{u}}$ state (HOMO) decreases significantly, while that of the $1^{2} \mathrm{~B}_{1 \mathrm{u}}$ state (next-HOMO) changes only slightly. In addition, the first and second ionized peaks observed at 6.9 and $7.2 \mathrm{eV}$ are underestimated by calculation $\mathrm{B}$ at 5.96 and $6.08 \mathrm{eV}$, as with calculation $\mathrm{C}$, despite the use of polarization d-functions. This could be due to the fixed $D_{4 h}$ structure or poor primitive basis sets.
TABLE 6: Ionized States of Free-Base Porphin by SAC-CI Calculations $B$ and $C$

\begin{tabular}{|c|c|c|c|c|c|c|}
\hline \multirow[b]{2}{*}{ state } & \multirow{2}{*}{$\begin{array}{l}\text { main configuration }^{a} \\
\qquad(|C|>0.3)\end{array}$} & \multirow[b]{2}{*}{ nature } & \multirow{2}{*}{$\begin{array}{c}\operatorname{exptl}^{b} \\
(\mathrm{eV})\end{array}$} & \multicolumn{3}{|c|}{$\begin{array}{c}\text { SAC-CI calculations } \\
(\mathrm{eV})\end{array}$} \\
\hline & & & & B & $\mathrm{C}$ & $\Delta^{c}$ \\
\hline $1^{2} \mathrm{~A}_{\mathrm{u}}$ & $-0.98(57)$ & $\pi$ & 6.9 & 5.96 & 6.35 & -0.39 \\
\hline $1^{2} \mathrm{~B}_{1 \mathrm{u}}$ & $0.98(56)$ & $\pi$ & $7.2 \mathrm{sh}$ & 6.08 & 6.09 & -0.01 \\
\hline $1^{2} \mathrm{~B}_{3 \mathrm{~g}}$ & $0.98(55)$ & $\pi$ & 8.4 & 7.91 & 7.98 & -0.07 \\
\hline $2^{2} \mathrm{~B}_{1 \mathrm{u}}$ & $-0.97(54)$ & $\pi$ & & 8.13 & 8.21 & -0.08 \\
\hline $1^{2} \mathrm{~A}_{\mathrm{g}}$ & $-0.97(49)$ & $\mathrm{n}$ & $8.8 \mathrm{sh}$ & 8.88 & 8.65 & +0.23 \\
\hline $1^{2} B_{2 g}^{g}$ & $0.77(53)-0.61(51)$ & $\pi$ & & 8.97 & 9.10 & -0.13 \\
\hline $1^{2} B_{2 u}$ & $-0.97(48)$ & $\mathrm{n}$ & 9.11 & 9.00 & 8.79 & +0.21 \\
\hline $3^{2} \mathrm{~B}_{1 \mathrm{u}}$ & $0.97(50)$ & $\pi$ & & 9.29 & 9.34 & -0.05 \\
\hline $2^{2} B_{2 g}$ & $0.77(51)+0.61(53)$ & $\pi$ & & 9.33 & 9.42 & -0.09 \\
\hline $2^{2} B_{3 g}$ & $0.98(52)$ & $\pi$ & & 9.37 & 9.54 & -0.17 \\
\hline $2^{2} \mathrm{~A}_{\mathrm{u}}$ & $0.98(47)$ & $\pi$ & 10.17 & 10.70 & 10.85 & -0.15 \\
\hline $1^{2} B_{1 g}$ & $-0.97(46)$ & $\sigma$ & 10.67 & 11.25 & 12.40 & -1.15 \\
\hline $1^{2} \mathrm{~B}_{3 \mathrm{u}}$ & $-0.94(45)$ & $\sigma$ & & 11.37 & 12.43 & -1.06 \\
\hline $2^{2} \mathrm{~B}_{2 \mathrm{u}}$ & $-0.88(44)+0.39(39)$ & $\sigma$ & 11.83sh & 11.85 & 12.77 & -0.92 \\
\hline $2^{2} B_{1 g}$ & $0.96(42)$ & $\sigma$ & & 11.90 & 12.67 & -0.77 \\
\hline $2^{2} B_{3 u}$ & $-0.95(41)$ & $\sigma$ & & 11.98 & 12.69 & -0.71 \\
\hline $2^{2} \mathrm{~A}_{\mathrm{g}}$ & $-0.96(38)$ & $\sigma$ & & 12.27 & 12.83 & -0.56 \\
\hline $3^{2} \mathrm{~B}_{2 \mathrm{u}}$ & $0.89(39)+0.40(44)$ & $\sigma$ & & 12.30 & 13.54 & -1.24 \\
\hline $3^{2} \mathrm{~A}_{\mathrm{g}}$ & $0.95(43)$ & $\sigma$ & 12.5 & 12.57 & 14.34 & -1.77 \\
\hline $4^{2} \mathrm{~B}_{1 \mathrm{u}}$ & $-0.97(40)$ & $\pi$ & & 12.63 & 12.74 & -0.11 \\
\hline $3^{2} \mathrm{~B}_{3 g}$ & $-0.97(37)$ & $\pi$ & & 13.06 & 13.19 & -0.13 \\
\hline $3^{2} B_{1 g}$ & $0.95(36)$ & $\sigma$ & & 13.43 & 14.16 & -0.73 \\
\hline $3^{2} \mathrm{~B}_{3 \mathrm{u}}$ & $-0.96(3$ & $\sigma$ & & 13.66 & 14.52 & -0.86 \\
\hline $4^{2} \mathrm{~B}_{2 \mathrm{u}}$ & $-0.97(3$ & $\sigma$ & 13.8 & 13.75 & 14.51 & -0.76 \\
\hline $4^{2} B_{1 g}$ & $-0.95(30)$ & $\sigma$ & & 13.94 & 14.62 & -0.68 \\
\hline $4^{2} \mathrm{~A}_{\mathrm{g}}$ & $0.95(32)$ & $\sigma$ & & 14.01 & 15.11 & -1.10 \\
\hline $3^{2} \mathrm{~B}_{2 g}$ & $0.97(33)$ & $\pi$ & & 14.06 & 14.13 & -0.07 \\
\hline $5^{2} B_{1 u}$ & $-0.97(31)$ & $\pi$ & & 14.18 & 14.25 & -0.07 \\
\hline $4^{2} \mathrm{~B}_{3 \mathrm{u}}$ & $-0.97(29)$ & $\sigma$ & & 14.88 & 15.27 & -0.39 \\
\hline $5^{2} \mathrm{~A}_{g}$ & $-0.97(28)$ & $\sigma$ & & 15.12 & 15.69 & -0.57 \\
\hline $5^{2} B_{3 u}$ & $0.96(27)$ & $\sigma$ & & 15.21 & 16.89 & -1.68 \\
\hline $5^{2} B_{2 u}$ & $0.95(2$ & $\sigma$ & 15.6 & 15.64 & 16.81 & -1.17 \\
\hline $6^{2} \mathrm{~A}_{\mathrm{g}}$ & $0.97(2$ & $\sigma$ & & 16.53 & 17.03 & -0.50 \\
\hline $6^{2} B_{2 u}$ & $0.95(24)$ & $\sigma$ & & 16.71 & 17.49 & -0.78 \\
\hline $5^{2} B_{1 g}$ & $0.96(23)$ & $\sigma$ & & 17.17 & 18.52 & -1.35 \\
\hline $6^{2} \mathrm{~B}_{3 \mathrm{u}}$ & $0.97(22)$ & $\sigma$ & & 17.78 & 18.72 & -0.94 \\
\hline $6^{2} B_{1 g}$ & $-0.96(21)$ & $\sigma$ & & 18.01 & 19.49 & -1.48 \\
\hline $7^{2} \mathrm{~A}_{\mathrm{g}}$ & $0.95(20)$ & $\sigma$ & & 18.29 & 19.40 & -1.11 \\
\hline $7^{2} \mathrm{~B}_{2 \mathrm{u}}$ & $-0.97(19)$ & $\sigma$ & & 18.77 & 19.73 & -0.96 \\
\hline $7^{2} B_{3 u}$ & $-0.97(18)$ & $\sigma$ & & 19.38 & 20.00 & -0.62 \\
\hline $7^{2} \mathrm{~B}_{1 \mathrm{~g}}$ & $-0.96(17)$ & $\sigma$ & & 19.53 & 20.87 & -1.34 \\
\hline $8^{2} \mathrm{~A}_{\mathrm{g}}$ & $0.96(16)$ & $\sigma$ & & 19.72 & 20.52 & -0.80 \\
\hline
\end{tabular}

${ }^{a}$ Numbering of the orbitals is given in Table 2 and Figure 2. ${ }^{b}$ Reference 28. ${ }^{c} \Delta$ is the difference between SAC-CI calculations B and $\mathrm{C}$.

The ionization energies of the $1^{2} \mathrm{~A}_{\mathrm{g}}$ and $1^{2} \mathrm{~B}_{2 \mathrm{u}}$ states with $\mathrm{n}$ nature increase and reflect the change in $\mathrm{MO}$ energies as described in section III. Furthermore, the peaks at $10 \sim 11 \mathrm{eV}$ are reassigned. The energy of the $2^{2} \mathrm{~B}_{3 \mathrm{~g}}$ state, $9.54 \mathrm{eV}$ in calculation $\mathrm{C}$, which was assigned to the experimental lower peak at $10.17 \mathrm{eV},{ }^{14}$ becomes even lower in calculation $\mathrm{B}, 9.37$ $\mathrm{eV}$, so that this state is reassigned to the higher side of the large peak at around $9 \mathrm{eV}$. The $2^{2} \mathrm{~A}_{\mathrm{u}}$ state with $\pi$ nature, $10.70 \mathrm{eV}$ in calculation $\mathrm{B}$, is then reassigned to the experimental lower peak, $10.17 \mathrm{eV}$. In addition, the energies of the higher states, $1^{2} \mathrm{~B}_{1 \mathrm{~g}}$ and $1^{2} \mathrm{~B}_{3 \mathrm{u}}$ with $\sigma$ nature, decrease about $1 \mathrm{eV}$ compared to those with calculation $\mathrm{C}$, so that these two states, 11.25 and $11.37 \mathrm{eV}$, are assigned to the experimental higher broad peak at $10.67 \mathrm{eV}$. This tendency is also seen with the other higher $\sigma$ states: the reorganization occurs in the higher energy region. Overall, the description of the ionization spectrum is improved by an addition of the polarization functions.

We here performed SAC-CI SD-R calculations, in which only single and double-excitation operators were included in the SAC-CI linked operators. This level of calculation is known 
good for the main peaks of the ionization spectra. ${ }^{43}$ However, for shake-up peaks, which involve two- and even higher electron processes, ${ }^{35,44,45}$ SAC-CI calculations using general-R operators (SAC-CI general-R) should be performed, ${ }^{44,45}$ though at the present stage, the computer code we have now is not efficient enough for this type of calculation. This is an interesting calculation to be done in future.

\section{Conclusions}

The ground, excited, and ionized states of FBP were recalculated by the SAC/SAC-CI method with adding Rydberg $\mathrm{s}$ and $\mathrm{p}$ functions on all of the nitrogen atoms (calculation $\mathrm{A}$ ) and with adding polarization d-functions on all of the nitrogen and carbon atoms (calculation B). The lowest Rydberg excited state appeared at around 5.5-5.8 eV with a weak intensity, but the valence-Rydberg mixing was small. The effects of the polarization and Rydberg functions were relatively small for the excitation energies of the main peaks, whereas the polarization functions affected rather largely the ionization spectrum. Therefore, the previous calculation $\mathrm{C}$ with $2 \mathrm{p}$ double $-\zeta$ basis sets was valid for the excited states but not enough for the ionized states.

Acknowledgment. This study was supported in part by a Grant-in-Aid for Scientific Research from the Japanese Ministry of Education, Science, and Culture and by the New Energy and Industrial Technology Development Organization (NEDO).

\section{References and Notes}

(1) Gouterman, M. The porphyrins; Dolphin, D., Ed.; Academic: New York, 1977; Vol. III.

(2) Weiss, C.; Kobayashi, H.; Gouterman, M. J. Mol. Spectrosc. 1965, $16,415$.

(3) Sundbom, M. Acta Chim. Scand. 1968, 22, 1317.

(4) McHugh, J. A.; Gouterman, M.; Weiss, C. Theor. Chim. Acta 1972, 24,346 .

(5) Sekino, H.; Kobayashi, H. J. Chem. Phys. 1987, 86, 5045.

(6) Edwards, W. D.; Weiner, B.; Zerner, M. C. J. Am. Chem. Soc. 1986, 108, 2196.

(7) Sekino, H.; Kobayashi, H. J. Chem. Phys. 1981, 75, 3477.

(8) Baker, J. D.; Zerner, M. C. Chem. Phys. Lett. 1990, 175, 192.

(9) Petke, J. D.; Maggiora, G. M.; Shipman, L. L.; Christoffersen, R. E. J. Mol. Spectrosc. 1978, 71, 64. (10).

(11) Foresman, J. B.; Head-Gordon, M.; Pople, J. A.; Frisch, M. J. J. Phys. Chem. 1992, 96, 135.

(12) Yamamoto, Y.; Noro, T.; Ohno, K. Int. J. Quantum Chem. 1992, 42, 1563.
(13) Merchán, M.; Ortí, E.; Roos, B. O. Chem. Phys. Lett. 1994, 226,

(14) Nakatsuji, H.; Hasegawa, J.; Hada, M. J. Chem. Phys. 1996, 104 2321

(15) Hasegawa, J.; Hada, M.; Nonoguchi, M.; Nakatsuji, H. Chem. Phys. Lett. 1996, 250, 159.

(16) Toyota, K.; Hasegawa, J.; Nakatsuji, H. Chem. Phys. Lett. 1996, 250, 437.

(17) Toyota, K.; Hasegawa, J.; Nakatsuji, H. J. Phys. Chem. A 1997, 101, 446.

(18) Nakatsuji, H.; Hasegawa, J.; Ueda, H.; Hada, M. Chem. Phys. Lett. 1996, 250, 379.

(19) Nakatsuji, H.; Tokita, Y.; Hasegawa, J.; Hada, M. Chem. Phys. Lett. 1996, 256, 220 .

(20) Tokita, Y.; Nakatsuji, H. J. Phys. Chem. B 1997, 101, 3281.

(21) Almlöf, J. Int. J. Quantum Chem. 1974, 3, 915.

(22) Suntum, S. F.; Case, D. A.; Karplus, M. J. Chem. Phys. 1983, 79, 7881.

(23) Merchán, M.; Ortí, E.; Roos, B. O. Chem. Phys. Lett. 1994, 221, 136.

(24) Yamamoto, S.; Kashiwagi, H. Chem. Phys. Lett. 1993, 205, 306.

(25) Yamamoto, S.; Teraoka, J.; Kashiwagi, H. J. Chem. Phys. 1988, $88,303$.

(26) Edwards, L.; Dolphin, D. H. J. Mol. Spectrosc. 1971, 38, 16.

(27) Dupuis, P.; Roberge, R.; Sandorfy, C. Chem. Phys. Lett. 1980, 75 , 434.

(28) Nakatsuji, H.; Hirao, K. J. Chem. Phys. 1978, 68, 2035.

(29) Nakatsuji, H. Chem. Phys. Lett. 1978, 59, 362.

(30) Nakatsuji, H. Chem. Phys. Lett. 1979, 67, 329, 334.

(31) Nakatsuji, H. J. Chem. Phys. 1984, 80, 3703.

(32) Nakatsuji, H.; Kitao, O.; Yonezawa, T. J. Chem. Phys. 1985, 83, 723.

(33) Kitao, O.; Nakatsuji, H. J. Chem. Phys. 1987, 87, 1169.

(34) Robin, M. B. Higher Excited States of Polyatomic Molecules; Academic: New York, 1975; Vol. I

(35) Nakatsuji, H. In Computational Chemistry-Reviews of Recent trends, Vol. 2: SAC-CI Method: Theoretical Aspects and Some Recent Topics; Leszczynski, J., Ed.; World Scientific: Singapore, 1996.

(36) Nakatsuji, H. Acta Chim. Hung. 1992, 129, 719.

(37) Huzinaga, S.; Andzelm, J.; Klobukowski, M.; Radzio-Andzelm, E.; Sakai, E.; Takewaki, H. Gaussian basis set for molecular calculation; Elsevier: New York, 1984

(38) Huzinaga, S. J. Chem. Phys. 1965, 42, 1293.

(39) Nakatsuji, H. Program system for SAC and SAC-CI calculations, Program Library No. 146 (Y4/SAC), Data Processing Center of Kyoto University, 1985; Program Library SAC85, No. 1396, Computer Center of the Institute for Molecular Science, Okazaki, 1981.

(40) Nakatsuji, H.; Hada, M.; Ehara, M.; Hasegawa, J.; Nakajima, T.; Nakai, H.; Kitao, O.; Toyota, K. The modified version of SAC85, to be published.

(41) Nakatsuji, H. Chem. Phys. 1983, 75, 425.

(42) Nakatsuji, H.; Musher, J. I. Chem. Phys. Lett. 1974, 24, 77.

(43) Nakatsuji, H.; Ohta, K.; Yonezawa, T. J. Phys. Chem. 1983, 87 3068

(44) Nakatsuji, H. Chem. Phys. Lett. 1991, 177, 331.

(45) Ehara, M.; Nakatsuji, H. Chem. Phys. Lett., in press. 